\title{
Fourier's Law in a Quantum Spin Chain and the Onset of Quantum Chaos
}

\author{
Carlos Mejía-Monasterio ${ }^{(a)}$, Tomaž $\operatorname{Prosen}^{(b)}$, and Giulio Casati ${ }^{(a, c, d)}$ \\ ${ }^{(a)}$ Center for Nonlinear and Complex Systems, Università degli Studi dell'Insubria, via Valleggio 11, Como 22100, Italy \\ ${ }^{(b)}$ Physics Department, Faculty of Mathematics and Physics, University of Ljubljana, Ljubljana, Slovenia \\ ${ }^{(c)}$ Istituto Nazionale per la Fisica della Materia, Unità di Como and \\ ${ }^{(d)}$ Istituto Nazionale di Fisica Nucleare, Sezione di Milano
}

(Dated: November 13, 2018)

\begin{abstract}
We study heat transport in a nonequilibrium steady state of a quantum interacting spin chain. We provide clear numerical evidence of the validity of Fourier law. The regime of normal conductivity is shown to set in at the transition to quantum chaos.

PACS numbers: 05.30.-d, 05.70.Ln, 05.45.Mt
\end{abstract}

The derivation of Fourier's law of heat conduction from the microscopic dynamics, without any ad hoc statistical assumption, is one of the great challenges of nonequilibrium statistical mechanics [1]. Even in the context of classical dynamical systems, the issue of energy (heat) transport, in spite of having a long history (recently reviewed in [2]), is not completely settled. It has become clear that transport theory requires that the underlying deterministic dynamics yield a truly diffusive process. On the other hand, it is known that classical nonlinear systems of interacting particles, above a critical interaction strength, typically exhibit chaotic and diffusive behaviour which then leads to the onset of Fourier law $J=-\kappa \nabla T$, relating the macroscopic heat flux to the temperature gradient $\nabla T$. Therefore deterministic chaos appears to be an essential ingredient required by transport theory. In this perspective, of particular interest is the problem, almost completely unexplored, of the derivation of Fourier law from quantum dynamics. So far, investigations have been mainly focused on linear response theory [5, 6, 7, 8]. The possibility instead to derive the Fourier law from quantum dynamics, by establishing the dependence of $J$ on $\nabla T$ in a nonequilibrium steady state, calls directly in question the issue of quantum chaos. In this connection, a main feature of quantum motion is the lack of exponential dynamical instability [9], a property which is at the heart of classical dynamical chaos. This fact may render very questionable the possibility to derive the Fourier law of heat conduction in quantum mechanics. However, quite interestingly, it has been shown that strong, exponential unstable, classical chaos is not necessary [3] (actually, strictly speaking, is not even sufficient [4]) for normal transport. Thus it is interesting to inquire if, and under what conditions, Fourier law emerges from the laws of quantum mechanics. This is the purpose of the present paper.

To investigate this problem one has to deal with a finite open system connected to heat baths. Here we consider an interacting quantum spin- $1 / 2$ chain which exhibits the transition from integrability to quantum chaos as a parameter, e.g. the magnetic field, is varied. The standard treatment of this problem is based on the master equation, thus limiting investigations to relatively small system sizes. By using this method, in an interesting paper [10], the decay of current correlation function in a model of non-integrable chain of quantum spins is computed. Here we take a different approach namely we follow the evolution of the system described by a pure state, which is stochastically coupled to an idealized model of heat baths. Stochastic coupling is realized in terms of a local measurement at the boundary of the system and stochastic but unitary exchange of energy between the system and the bath. By this method we have been able to perform very effective numerical simulations which allow to observe a clear energy/temperature profile and to measure the heat current $J$. In the nonintegrable regime where the spectral statistics is described by random matrix theory (RMT) - the regime of "quantum chaos" - we found very accurate Fourier law scaling $J / \Delta T \propto 1 / L$, where $L$ is the size of the chain. In the integrable and near-integrable regimes instead, we found that the heat transport is ballistic $J \propto L^{0}$.

We consider an Ising chain of $L$ spins $1 / 2$ with coupling constant $Q$ subject to a uniform magnetic field $\vec{h}=\left(h_{x}, 0, h_{z}\right)$, with open boundaries. The Hamiltonian reads

$$
\mathcal{H}=-Q \sum_{n=0}^{L-2} \sigma_{n}^{z} \sigma_{n+1}^{z}+\vec{h} \cdot \sum_{n=0}^{L-1} \vec{\sigma}_{n}
$$

where the operators $\vec{\sigma}_{n}=\left(\sigma_{n}^{x}, \sigma_{n}^{y}, \sigma_{n}^{z}\right)$ are the Pauli matrices for the $n$-th spin, $n=0,1, \ldots L-1$. We set the coupling constant $Q=2$. In this system, the only trivial symmetry is a reflection symmetry, $\vec{\sigma}_{n} \rightarrow \vec{\sigma}_{L-1-n}$. Moreover the direction of the magnetic field affects the qualitative behavior of the system: If $h_{z}=0$, the Hamiltonian (11) corresponds to the Ising chain in a transversal magnetic field. In this case the system is integrable as (1) can be mapped into a model of free fermions through standard Wigner-Jordan transformations. When $h_{z}$ is increased from zero, the system is no longer integrable. When $h_{z}$ is of the same order of $h_{x}$ quantum chaos sets in leading to a very complex structure of quantum states as well as to fluctuations in the spectrum that are statistically described by RMT[1]. The system becomes 


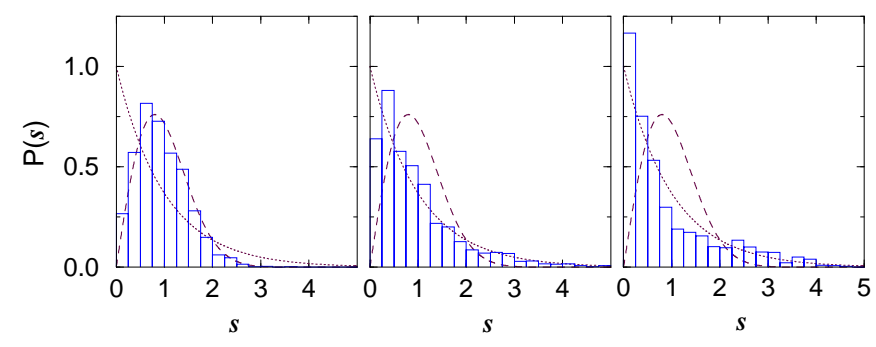

FIG. 1: Nearest neighbor level spacing distribution $P(s)$ for the chaotic (left), intermediate (center) and integrable (right) chains. $P(s)$ was obtained by diagonalizing the Hamiltonian (1) for a chain of length $L=12$, averaging over even and odd parity subspectra. The curves correspond to $P_{\mathrm{WD}}$ (dashed line) and to $P_{\mathrm{P}}$ (dotted line).

again (nearly) integrable when $h_{z} \gg h_{x}$. Therefore, by choosing the direction of the external field we can explore different regimes of quantum dynamics.

The onset of quantum chaos is commonly studied in terms of the nearest neighbor level spacing distribution $P(s)$ that gives the probability density to find two adjacent levels at a distance $s$. For an integrable system the distribution $P(s)$ has typically a Poisson distribution $P_{\mathrm{P}}(s)=\exp (-s)$. In contrast, in the quantum chaos regime (for Hamiltonians obeying time-reversal invariance), $P(s)$ is given by the Gaussian Orthogonal Ensemble of random matrices (GOE). In this case, $P(s)$ is well-approximated by the Wigner surmise $P_{\mathrm{WD}}(s)=$ $(\pi s / 2) \exp \left(-\pi s^{2} / 4\right)$, exhibiting the so-called "level repulsion".

In Fig. 1 we show the results of our numerical simulations of system (11) for three different values of the magnetic field: (i) chaotic case $\vec{h}=(3.375,0,2)$ at which the distribution $P(s)$ agrees with GOE and thus corresponds to the regime of quantum chaos, (ii) integrable case $\vec{h}=(3.375,0,0)$, at which $P(s)$ is close to the Poisson distribution, and (iii) intermediate case $\vec{h}=(7.875,0,2)$ at which the distribution $P(s)$ shows a combination of (weak) level repulsion and an exponential tail. We have consistently observed signatures for the onset of quantum chaos also in other spectral correlations as well as in the structure of eigenfunctions.

Let us now turn to study energy transport in this model system. To this end we need to couple both ends of the chain of spins to thermal reservoirs at temperature $T$. We have devised a simple way to simulate this coupling, namely the state of the spin in contact with the bath is statistically determined by a Boltzmann distribution with parameter $T$. Our model for the reservoirs is analogous to the stochastic thermal reservoirs used in classical simulations 12 and we thus call it a quantum stochastic reservoir. We use units in which Planck and Boltzmann constants are set to unity $\hbar=k_{\mathrm{B}}=1$.
In the representation basis of $\sigma_{n}^{z}$ the wave function at time $t$ can be written as

$$
|\psi(t)\rangle=\sum_{s_{0}, s_{1}, \ldots, s_{L-1}} C_{s_{0}, s_{1}, \ldots, s_{L-1}}(t)\left|s_{0}, s_{1} \ldots s_{L-1}\right\rangle,
$$

where $s_{n}=0,1$ represents the up, down state of the $n$-th spin, respectively. The wave function at time $t$ is obtained from the unitary evolution operator $\mathrm{U}(t)=$ $\exp (-i \mathcal{H} t)$. The interaction with the reservoir is not included in the unitary evolution. Instead, we assume that the spin chain and the reservoir interact only at discrete times with period $\tau$ at which the states of the leftmost $\left(s_{0}\right)$ and the rightmost $\left(s_{L-1}\right)$ spins are stochastically reset. Thus, the evolution of the wave function from time $t$ to time $t+\tau$ can be represented as

$$
|\psi(t+\tau)\rangle=\Xi\left(\beta_{1}, \beta_{\mathrm{r}}\right) \mathrm{U}(\tau)|\psi(t)\rangle,
$$

where $\Xi\left(\beta_{1}, \beta_{\mathrm{r}}\right)$ represents the unitary stochastic action of the interaction with the left and right reservoirs at temperatures $\beta_{1}^{-1}$ and $\beta_{\mathrm{r}}^{-1}$ respectively.

The action of $\Xi\left(\beta_{1}, \beta_{\mathrm{r}}\right)$ takes place in several steps:

(i) The wave function is first rotated by the angle $\alpha=\tan ^{-1}\left(h_{x} / h_{z}\right)$ to the eigenbasis of the components $\sigma_{\mathrm{l}}=\vec{h} \cdot \vec{\sigma}_{0} / h, \sigma_{\mathrm{r}}=\vec{h} \cdot \vec{\sigma}_{L-1} / h$ of the edge spins along the field, that is $|\psi\rangle \rightarrow e^{-i \alpha\left(\sigma_{0}^{y}+\sigma_{L-1}^{y}\right) / 2}|\psi\rangle$. Here, $h=|\vec{h}|$ stands for the strength of the magnetic field.

(ii) A local measurement of the observables $\sigma_{1}, \sigma_{\mathrm{r}}$ is performed. Then the state of the spins at the borders collapses to a state $\left(s_{0}^{*}, s_{L-1}^{*}\right)$ with probability

$$
p\left(s_{0}^{*}, s_{L-1}^{*}\right)=\sum_{s_{1}, \ldots, s_{L-2}}\left|C_{s_{0}^{*}, s_{1}, \ldots, s_{L-2}, s_{L-1}^{*}}\right|^{2} .
$$

In other words this means that we put all coefficients $C_{s_{0}, s_{1}, \ldots, s_{L-1}}$ with $\left(s_{0}, s_{L-1}\right) \neq\left(s_{0}^{*}, s_{L-1}^{*}\right)$ to zero.

(iii) The new state of the edge spins is stochastically chosen. After this, which simulates the thermal interaction with the reservoirs, each of the edge spins is set to down, (up) state with propability $\mu,(1-\mu)$. The probability $\mu(\beta)$ depends on the canonical temperature of each of the thermal reservoirs:

$$
\mu\left(\beta_{j}\right)=\frac{e^{\beta_{j} h}}{e^{-\beta_{j} h}+e^{\beta_{j} h}} \quad ; \quad j \in\{\mathrm{l}, \mathrm{r}\} .
$$

(iv) Finally, the wave function is rotated back to the $\sigma_{n}^{z}$ basis, $|\psi\rangle \rightarrow e^{i \alpha\left(\sigma_{0}^{y}+\sigma_{L-1}^{y}\right) / 2}|\psi\rangle$.

This completes the description of the interaction with the quantum stochastic bath. This interaction thus (periodically) resets the value of the local energy $h \sigma_{1, \mathrm{r}}$ of the spins in contact with the reservoirs. This information is then transmitted to the rest of the system during its dynamical evolution and relaxation towards equilibirum. Therefore, the value of $\tau$ controls the strength of the coupling to the bath. We have found that, in our units, $\tau=1$ provides an optimal choice. We have nevertheless 


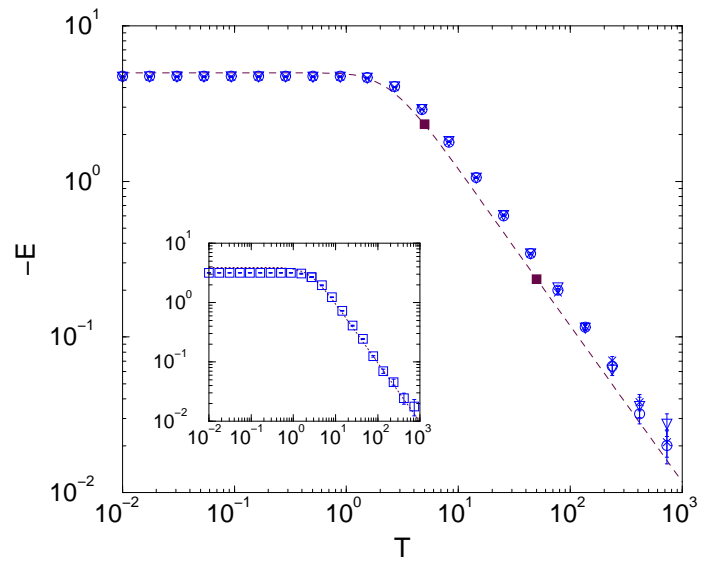

FIG. 2: Mean local energy $E$ as a function of the temperature $T$ for the chaotic chain, for $L=6,8,10$ with crosses, triangles and circles, respectively. Each point corresponds to the equilibrium simulation in which the bath temperatures are set to the same value $T$. The average was obtained from the center of the profile so that, the two sites closest to each border were not considered. The dashed curve corresponds to the canonical average $E_{\text {can }}(T)$ of the local 2-body energy operator $H_{n}$. The two solid squares indicate the values of the temperature that were later used in the out of equilibrium simulations. In the inset the same data are shown for the integrable chain (squares) for $L=8$.

performed simulations for other values of $\tau$ with qualitatively similar results.

The unitary evolution $U(t)|\psi\rangle$ of the system has been computed by an accurate high order split-step factorization of the unitary evolution operator [13]. For each run the initial wavefunction $|\psi(0)\rangle$ of the system is chosen at random. The system is then evolved for some relaxation time $\tau_{\text {rel }}$ after which it is assumed to fluctuate around a unique steady state. Measurements are then performed as time averages of the expectation value of suitable observables. We further average these quantities over different random realizations of "quantum trajectories".

In order to compute the energy profile we write Hamiltonian (11) in terms of local energy density operators $H_{n}$ :

$$
H_{n}=-Q \sigma_{n}^{z} \sigma_{n+1}^{z}+\frac{\vec{h}}{2} \cdot\left(\vec{\sigma}_{n}+\vec{\sigma}_{n+1}\right) .
$$

The local Hamiltonian $H_{n}$ (defined for $0<n<L-2$ ), gives the energy density between the $n$-th and $(n+1)$-th spins. In terms of eq. (6) the Hamiltonian of the system can be rewritten as

$$
\mathcal{H}=\sum_{n=0}^{L-2} H_{n}+\frac{h}{2}\left(\sigma_{1}+\sigma_{\mathrm{r}}\right) .
$$

First we have performed equilibrium simulations in order to show that time averaged expectation values of the local energy density can be used as a consistent canonical local temperature. To this end we set the left and

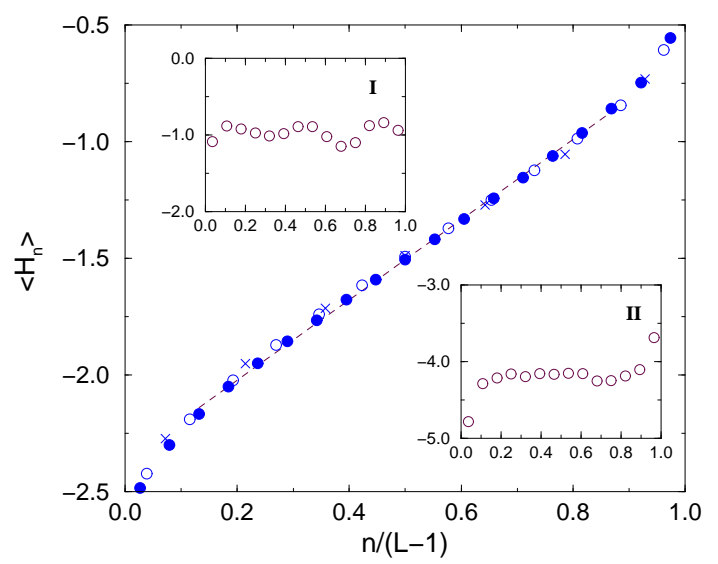

FIG. 3: Energy profile $\left\langle H_{n}\right\rangle$ for an out of equilibrium situation for the chaotic chain. The nominal values of the temperatures of the reservoirs $T_{1}=5$ and $T_{\mathrm{r}}=50$, are both in the high temperature regime (see the solid squares in Fig. 20 The different symbols correspond to chains of size $L=8$ (crosses), $L=14$ (open circles) and $L=20$ (solid circles). The dashed line was obtained from a linear fit of the data for $L=20$ in which the two sites closest to each border were not considered. In the insets (I) and (II) the energy profile is shown for the integrable and intermediate cases respectively, for $L=15$.

right reservoirs to the same temperature $T$. In Fig. 22 we plot the mean local energy $E=\left\langle H_{n}\right\rangle$ where the average is taken over time and over the $L-5$ central values of the profile, for the chaotic and for the integrable chain (inset). For low $T, E$ saturates to a constant which, together with the energy profile $\left\langle H_{n}\right\rangle$, is determined by the ground state. However, for larger $T>1$, the energy profile is constant within numerical accuracy, and numerical simulations give $E \sim-1 / T$, all results being almost independent of $L$ for $L \geq 6$. The numerical data for $E(T)$ can be well approximated with a simple calculation of energy density for a two-spin chain $(L=2)$ in a canonical state at temperature $T$ (smooth curves), namely $E_{\text {can }}(T)=\operatorname{tr} H_{0} e^{-H_{0} / T} / \operatorname{tr} e^{-H_{0} / T}$. Therefore, if the temperatures of both reservoirs are in high $T$ regime, then we can define the local temperature via the relation $T \propto-1 / E$. We stress that equilibrium numerical data shown are insensitive to the nature of dynamics (consistent with results of Ref.[14]), whether being chaotic, regular or intermediate.

In Fig. 3 we show the energy profile $\left\langle H_{n}\right\rangle$ for an out of equilibrium simulation of the chaotic chain. In all nonequilibrium simulations, the temperatures of the baths were set to $T_{1}=5$ and $T_{\mathrm{r}}=50$. After an appropriate scaling the profiles for different sizes collapse to the same curve. More interesting, in the bulk of the chain the energy profile is in very good approximation linear. In contrast, we show that in the case of the integrable (inset I) and intermediate (inset II) chains, no energy gradient is created. 


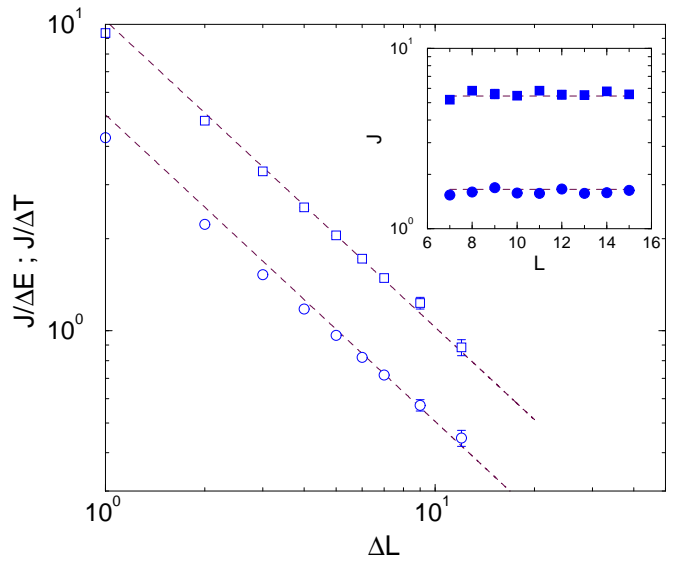

FIG. 4: Size dependence of the energy current in the chaotic chain with $T_{1}=5$ and $T_{\mathrm{r}}=50$. We show $J / \Delta E$ (open circles) and $J / \Delta T$ (open squares). The dashed lines corresponds to $1 / \Delta L$ scaling. In the inset, the size dependence of the energy current is shown for the integrable (solid circles) and the intermediate (solid squares).

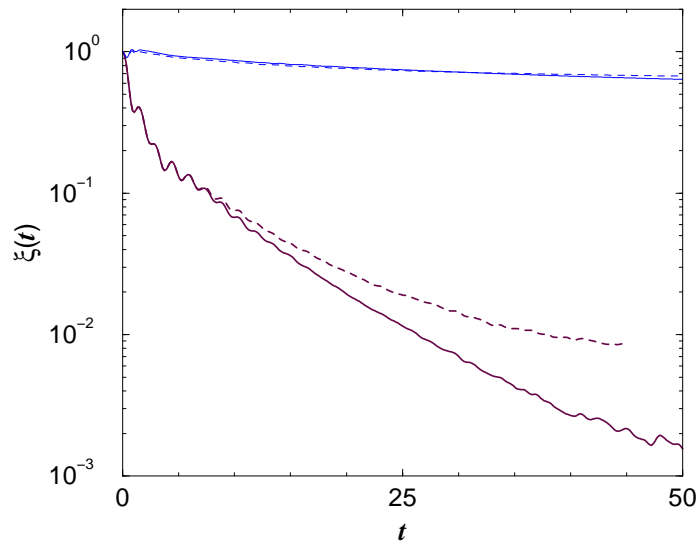

FIG. 5: Infinite temperature current-current time correlation function $\xi(t)$, for two chaotic chains of size $L=18$ (tick dashed line) and $L=24$ (thick solid line) and for two integrable chains of size $L=18$ (dashed line) and $L=24$ (solid line).

We now define the local current operators through the equation of continuity: $\partial_{t} H_{n}=i\left[\mathcal{H}, H_{n}\right]=-\left(J_{n+1}-J_{n}\right)$, requiring that $J_{n}=\left[H_{n}, H_{n-1}\right]$. Using eqs. (6) and (7) the local current operators are explicitly given by

$$
J_{n}=h_{x} Q\left(\sigma_{n-1}^{z}-\sigma_{n+1}^{z}\right) \sigma_{n}^{y}, \quad 1 \leq n \leq L-2 .
$$

In Fig. 4 we plot $J / \Delta E$ as a function of the size $L$ of the system for sizes up to $L=20$. The mean current $J$ is calculated as an average of $\left\langle J_{n}\right\rangle$ over time and $L-8$ central values of $n$. The energy difference was obtained from the energy profile as $\Delta E=\left\langle H_{L-5}\right\rangle-\left\langle H_{3}\right\rangle$. Three spins near each bath have been discarded in order to be in the bulk regime. Since $\Delta L=L-8$ is an effective size of the truncated system, the observed $1 / \Delta L$ dependence confirms that the transport is normal. Moreover, also the quantity $J / \Delta T$, where $\Delta T=-1 /\left\langle H_{L-5}\right\rangle+1 /\left\langle H_{3}\right\rangle$, shows the correct scaling with the size $L$. On the other hand, in integrable and intermediate chains we have observed that the average heat current does not depend on the size $J \propto L^{0}$, clearly indicating a ballistic transport.

As a consistency check, we have also studied the energy current-current time correlation $\xi(t)=\langle J(t) J\rangle$ of the isolated system for $\beta \rightarrow 0$ which, for high temperature, gives the conductivity as $\kappa=\beta L \int_{0}^{\infty} \xi(t) \mathrm{d} t$. In Fig. [5 we show that for the chaotic chain $\xi(t)$ exhibits fast, possibly exponential decay while for the intermediate case we observe slow decay, possibly to a finite plateau, giving rise to ballistic transport. In the integrable case $\xi(t)$ is constant since $J(t)$ is a conserved quantity.

In this paper we have shown that Fourier law of heat conduction can be derived from the pure quantum dynamical evolution without any additional statistical assumption. This possibility is strictly related to the onset of quantum chaotic behavior.

TP acknowledges financial support by the grant P10044 of the Ministry of Science and Technology of Slovenia.

[1] F. Bonetto, J.L. Lebowitz and L. Rey-Bellet in Mathematical Physics 2000, A. Fokas, A. Grigoryan, T. Kibble and B. Zegarlinsky (Eds.), Imperial College London, 128 (2000).

[2] S. Lepri, R. Livi and A. Politi Phys.Rep. 377, 1 (2003).

[3] B. Li, G. Casati, J. Wang and T. Prosen, Phys. Rev. Lett. 92, 254301 (2004).

[4] S. Lepri, R. Livi and A. Politi, Phys. Rev. Lett. 78, 1896 (1997).

[5] R. Kubo, J. Phys. Soc. Jpn. 12, 570 (1957).

[6] X. Zotos and P. Prelovšek, cond-mat/0304630

[7] X. Zotos, F. Naef and P. Prelovšek, Phys. Rev. B, 55, 11029 (1997)

[8] T. Prosen, Phys. Rev. Lett., 88, 1808 (1998)

[9] G. Casati, B. V. Chirikov, I. Guarneri and D. L. Shepelyansky, Phys. Rev. Lett. 56, 2437 (1986).

[10] K. Saito, Europhys. Lett. 61, 34 (2003).

[11] T. Guhr, A. Müller-Groeling, and H.A. Weidenmüller, Phys. Rep. 299, 189 (1998)

[12] H. Larralde, F. Leyvraz, C. Mejia-Monasterio J. Stat. Phys. 113, 197 (2003).

[13] On the basis of a general idea of M. Suzuki, Phys. Lett. A 165, 387 (1992), we have derived and used a very convenient complex-coefficient split-step formula for the short-time evolution $U(\epsilon)=e^{(A+B) \epsilon}=$ $e^{p_{1} A \epsilon} e^{p_{2} B \epsilon} e^{p_{3} A \epsilon} e^{p_{4} B \epsilon} e^{p_{5} A \epsilon}+\mathcal{O}\left(\epsilon^{4}\right)$, with $p_{1}=p_{5}^{*}=$ $(1+i / \sqrt{3}) / 4, p_{2}=p_{4}^{*}=2 p_{1}, p_{5}=1 / 2$.

[14] R. V. Jensen and R. Shankar, Phys. Rev. Lett. 54, 1879 (1985). 